\title{
CDMA Signature Sequences with Low Peak-to-Average-Power Ratio via Alternating Projection
}

\author{
Joel A. Tropp \\ Inst. for Comp. Engr. and Sci. (ICES) \\ The University of Texas at Austin \\ 1 University Station C0200 \\ Austin, TX 78712 \\ jtropp@ices.utexas.edu
}

\author{
Inderjit. S. Dhillon \\ Dept. of Comp. Sci. \\ The University of Texas at Austi \\ 1 University Station C0500 \\ Austin, TX 78712 \\ inderjitocs.utexas.edu
}

\author{
Robert W. Heath Jr. \\ Dept. of Elect. and Comp. Engr. \\ The University of Texas at Austin \\ 1 University Station $\mathrm{C} 0803$ \\ Austin, TX 78712-1084 USA \\ rheath@ece. utexas.edu
}

\author{
Thomas Strohmer \\ Dept. of Mathematics \\ University of California, Davis \\ Davis, CA 95616 USA \\ strohmer@math.ucdavis.edu
}

\begin{abstract}
Several algorithms have been proposed to construct optimal signature sequences that maximize the sum capacity of the uplink in a direct-spread synchronous code division multiple access (CDMA) system. These algorithms produce signatures with real-valued or complexvalued entries that generally have a large peak-to-average power ratio (PAR). This paper presents an alternating projection algorithm that can design optimal signature sequences that satisfy PAR side constraints. This algorithm converges to a fixed point, and these fixed points are partially characterized.
\end{abstract}

\section{INTRODUCTION}

Signature sequences that maximize the sum capacity in the uplink of direct-spread synchronous code division multiple access (CDMA) systems have been characterized in [1], [2], [3]. Except in special cases, these signatures are generally real- or complex-valued. Consequently, these signatures can possess practically undesirable properties such as a large peak-to-averagepower ratio (PAR).

The PAR of a signal measures how the largest value of the signal compares with the average power. Signals with large PAR require higher dynamic range on the analogto-digital converters and the digital-to-analog converters. They may also require more linear (and thus higher cost) power amplifiers. In DS-CDMA systems, the PAR is normally of concern only in the downlink (see e.g. [4]), where linear combinations of signatures can conspire to have very large PAR values. The problem of PAR on the

${ }^{1} 1$. S. Dhillon acknowledges support by NSF CAREER Award No. ACI-0093404, T. Strohmer acknowledges partial support by NSF grant DMS 0208568 , and R. Heath acknowledges support from the Texas Advanced Technology Program under Grant No. 003658 0614-2001, the Samsung Advanced Institute of Technology, and the National Instruments Foundation. uplink is fundamentally different because it only involves individual signatures. Conventionally, the PAR on the uplink has not received attention because uplink typically employs binary spreading sequences, which have unit PAR. If sum-capacity optimal sequences are to be used in real systems, however, PAR side constraints should be included in the design problem.

Several algorithms have been developed for construction of optimal sequences. Viswanath and Anantharam [2] offer a finite-step algorithm that can construct a limited selection of optimal CDMA sequences. A number of iterative algorithms, including [5], [6], [7], have been developed that can construct many more sequences than the finite-step algorithm. Unfortunately, these methods cannot accept additional constraints on the signatures, and thus are not suitable in general for finding sequences with low PAR.

In this paper, we give a new algorithm for finding optimal signature sequences with constraints on the PAR. Our algorithm is enabled by the observation that the class of optimal signatures is so large that we can impose additional constraints without losing the optimality. We build on our recently proposed iterative algorithm for constructing CDMA signature sequences [8]. These algorithms are related to a method used by Chu for solving an inverse eigenvalue problem [9]. We argue that our algorithm converges to a fixed point, and we claim that the class of fixed points contains the desired sequences. Proofs of these results will appear elsewhere [10].

\section{Problem Formulation}

Consider a direct-spread synchronous CDMA system with $N$ users and a processing gain of $d$, operating in the presence of white noise. Suppose that the average input power of the $n$-th user is denoted by $w_{n}$. We assume 
that no user is oversized [2], since the extension to this case is straightforward. A mathematical statement of this hypothesis is

$$
w_{n} \leq \frac{1}{d} \sum_{j=1}^{N} w_{j} \quad \text { for } \quad n=1, \ldots, N .
$$

A signature sequence is a collection $\left\{s_{n}\right\}$ of $N$ unitnorm vectors in $\mathbb{C}^{d}$. Define the weighted signatures $\boldsymbol{x}_{n} \stackrel{\text { d.f }}{=} \sqrt{w_{n}} s_{n}$, and form a $d \times N$ matrix $X$ whose $n$-th column is $\boldsymbol{x}_{n}$. For purposes of design, we consider the discrete-time peak-to-average ratio based on the sampled discrete-time system, which approximates the PAR of the continuous-time system. The PAR of a $d$-dimensional signature $v$ is defined as

$$
\operatorname{PAR}(v) \stackrel{\text { def }}{=} \frac{\max _{\ell}\left|v_{\ell}\right|^{2}}{d^{-1} \sum_{\ell}\left|v_{\ell}\right|^{2}} .
$$

We use $\rho$ to denote the desired upper bound on the PAR of the signatures. Note that $1 \leq \operatorname{PAR}(v) \leq d$. The lower extreme corresponds to a signature whose entries have identical modulus, while the upper bound is attained only by (scaled) canonical basis vectors.

Viswanath and Anantharam have shown that a signature sequence achieves the sum capacity of the present system model if and only if the $d$ positive singular values of $X$ are identical. A matrix with this property is called a tight frame. Our goal, then, is to construct a weighted signature matrix $X$ with the following properties.

i. The matrix is a tight frame: $X X^{*}=\alpha I_{d}$.

ii. Each column has the correct norm: $\left\|\boldsymbol{x}_{n}\right\|_{2}^{2}=w_{n}$.

iii. Each column has low PAR: $\operatorname{PAR}\left(x_{n}\right) \leq \rho$.

In this paper we present an algorithm that calculates such sequences. In the sequel, we summarize the method and its theoretical behavior.

\section{StATEMENT OF Algorithm}

Our technique is based on an alternating projection between Property (i) and Properties (ii)-(iii). The algorithm attempts to compute a nearby matrix (in terms of the Frobenius norm) that satisfies Properties (i)-(iii).

Algorithm I (Alternating Projection):

INPUT:

- An arbitrary matrix $S_{0}$

- The number of iterations $J$

OUtPut:

- A pair of matrices $\left(S_{J}, X_{J}\right)$

PRocedure:

1) Let $j=1$.

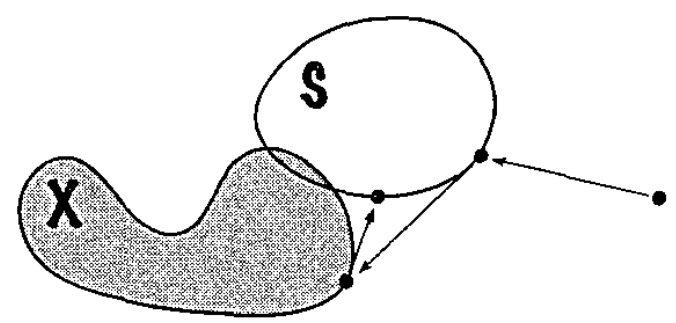

Fig. 1. Intuition behind the alternating projection between set $\mathscr{S}$ and $\mathfrak{X}$.

2) Find $X_{j}$, the matrix nearest to $S_{j-1}$ in Frobenius norm that has Property (i).

3) Find $S_{j}$, the nearest matrix to $X_{j}$ in Frobenius norm that has Properties (ii) and (iii).

4) Increment $j$. Repeat Steps 2-4 until $j>J$.

The intuition behind the operation of the alternating projection method is illustrated in Figure 1 . Notice in particular that the constraint sets are both closed but both are not necessarily convex.

The machinery of point-to-set maps is required to understand the convergence of this algorithm, so we must refer the reader to [10] for details. For reference, we shall state the convergence result. A few definitions are necessary. Let $\mathscr{S}$ be the collection of matrices that satisfy the structural properties (ii) and (iii). Let $\mathscr{X}$ be the collection of tight frames-matrices that satisfy property (i). Recall that the distance between a point $M$ and a set $\mathscr{Y}$ is

$$
\operatorname{dist}(M, \mathscr{Y})=\inf _{Y \in \mathscr{Y}}\|Y-M\|_{\mathrm{F}} .
$$

Theorem 2 (TDHS [10]): Suppose that alternating projection generates a sequence of iterates $\left\{\left(S_{j}, X_{j}\right)\right\}$. This sequence has at least one accumulation point, i.e. limit of a convergent subsequence.

- Every accumulation point lies in $\mathscr{S} \times \mathscr{X}$.

- Every accumulation point $(\bar{S}, \bar{X})$ satisfies

$$
\|\bar{S}-\bar{X}\|_{\mathrm{F}}=\lim _{j \rightarrow \infty}\left\|S_{j}-X_{j}\right\|_{\mathrm{F}}
$$

- Every accumulation point is a generalized fixed point, viz.

$$
\|\bar{S}-\bar{X}\|_{\mathrm{F}}=\operatorname{dist}(\bar{S}, \mathscr{X})=\operatorname{dist}(\bar{X}, \mathscr{S}) .
$$

We have been able to provide a partial characterization of the fixed points of this algorithm. It turns out the set of fixed points includes every collection of $N$ vectors that can be partitioned into tight frames for mutually orthogonal subspaces of $\mathbb{C}^{d}$. In particular, every matrix 


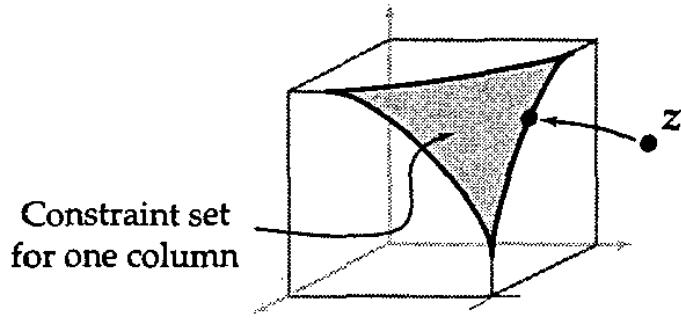

Fig. 2. The shaded region contains the vectors with squared norm $c$ that have PAR less than $\rho$. It equals the intersection of the sphere of radius $\sqrt{c}$ and the cube with sides $\sqrt{c \rho / d}$. The input vector to the neamess problem is $z$.

that satisfies Properties (i)-(iii) is a fixed point. The other fixed points are spurious solutions that rarely arise in practice.

Proposition 3 (TDHS [10]): Suppose that $S$ lies in $\mathscr{S}$ and that $S S^{*} S=S \Lambda$, where $\Lambda$ is positive and diagonal. Then $S$ is a (classical) fixed point of Algorithm 1. More precisely, invoking Algorithm 1 with the initial matrix $S$ will yield $S_{j}=S$ for every $j$.

The proof of this proposition appears in [10].

\section{IMPLEMENTATION}

To implement this algorithm, we must solve two matrix nearness problems. Step 2 is a standard problem from linear algebra, whose solution can be expressed in terms of a singular value decomposition [11]. If we factor $S_{j-1}=U \Sigma V^{*}$, then $X_{j}=(\operatorname{Tr} \Sigma / d) U V^{*}$ is a nearest tight frame to $S_{j-1}$. Here, $\operatorname{Tr}(\cdot)$ indicates the trace operator.

The nearest matrix to $X_{j}$ that satisfies the norm and peak-to-average-power criteria cannot be written in closed form. Fortunately, we can apply the following simple algorithm to each column $x_{n}$ of the input matrix to obtain $s_{n}$, the corresponding column of the output matrix $S_{j}$. See Figure 2 for a diagram of the constraint on each column.

Algorithm 4 (Nearest Vector with Low PAR): INPUT:

- An input vector $\boldsymbol{z}$ from $\mathbb{C}^{d}$

- A positive number $c$, the squared norm of the solution vector

- A number $\rho$ from $[1, d]$, which equals the maximum permissible PAR

\section{OUTPUT:}

- A vector $s$ from $\mathbb{C}^{d}$ that solves $\min _{\boldsymbol{s}}\|\boldsymbol{s}-\boldsymbol{z}\|_{2} \quad$ s.t. $\quad \operatorname{PAR}(s) \leq \rho$ and $\|s\|_{2}^{2}=c$.

\section{PROCEDURE:}

1) Scale $z$ to have unit norm; define $\delta=\sqrt{c \rho / d}$; and initialize $k=0$.

2) Let $\mathscr{M}$ index $(d-k)$ components of $z$ with least magnitude. If this set is not uniquely determined, increment $k$ and repeat Step 2.

3) If $z_{m}=0$ for each $m$ in $\mathscr{M}$, a solution vector is

$$
s= \begin{cases}\sqrt{\frac{c-k \delta^{2}}{d-k}} & \text { for } m \in \mathscr{M}, \text { and } \\ \delta \mathrm{e}^{\mathrm{i} \text { arg } z_{m}} & \text { for } m \notin \mathscr{M} .\end{cases}
$$

4) Otherwise, let

$$
\gamma=\sqrt{\frac{c-k \delta^{2}}{\sum_{m \in \mathscr{H}}\left|z_{m}\right|^{2}}} .
$$

5) If $\gamma z_{m}>\delta$ for any $m$ in $\mathscr{M}$, increment $k$ and return to Step 2 .

6) The unique solution vector is

$$
s= \begin{cases}\gamma z_{m} & \text { for } m \in \mathscr{M}, \text { and } \\ \delta \mathrm{e}^{\mathrm{i} \text { arg } z_{m}} & \text { for } m \notin \mathscr{M} .\end{cases}
$$

When $\rho=1$, the output of the algorithm. is a unimodular vector whose entries have the same phase as the corresponding entries of $\boldsymbol{z}$. On the other hand, when $\rho=d$, the output vector equals $z$. Let us prove that the algorithm is correct.

Proof. We must solve the optimization problem $\min _{s}\|s-z\|_{2}^{2} \quad$ subject to $\operatorname{PAR}(s) \leq \rho$ and $\|s\|_{2}^{2}=c$. Let us begin with some major simplifications. First, rewrite the PAR constraint by enforcing the norm requirement and rearranging to obtain the equivalent condition

$$
\max _{m}\left|s_{m}\right| \leq \sqrt{c \rho / d}
$$

In the rest of the argument, the symbol $\delta$ will abbreviate the quantity $\sqrt{c \rho / d}$. The PAR constraint becomes $\left|s_{m}\right| \leq \delta$ for each $m=1, \ldots, d$.

Now expand the objective function and enforce the norm constraint again to obtain

$$
\min _{\boldsymbol{s}}\left[c-2 \operatorname{Re}\langle s, z\rangle+\|z\|_{2}^{2}\right] .
$$

Observe that it is necessary and sufficient to minimize the second term. It follows that the optimizer does not depend on the scale of the input vector $z$. So take $\|z\|_{2}=$ 1 without loss of generality.

Next observe that the PAR constraint and the norm constraint do not depend on the phases of the components in $s$. Therefore, the components of an optimal $s$ must have the same phases as the components of the 
input vector $z$. In consequence, we may assume that both $\boldsymbol{s}$ and $\boldsymbol{z}$ are non-negative real vectors.

We have reached a much more straightforward optimization problem. Given a vector $\boldsymbol{z}$ with unit norm and non-negative entries, we must solve

$$
\max _{\boldsymbol{s}}\langle\boldsymbol{s}, \boldsymbol{z}\rangle \quad \text { subject to }\langle\boldsymbol{s}, \boldsymbol{s}\rangle=c \text { and } 0 \leq s_{m} \leq \delta .
$$

Observe that every point of the feasible set is a regular point. Therefore, Karush-Kuhn-Tucker theory will furnish necessary conditions on an optimizer [12].

We form the Lagrangian function

$$
\begin{aligned}
L(s, \lambda, \mu, \nu)=-\langle s, z\rangle+ & \frac{1}{2} \lambda(\langle s, s\rangle-c) \\
& -\langle s, \mu\rangle+\langle s-\delta 1, \nu\rangle .
\end{aligned}
$$

The Lagrange multipliers $\mu$ and $\nu$ are non-negative because they correspond to the lower and upper bounds on $s$. Meanwhile, the multiplier $\lambda$ is unrestricted because it is associated with the equality constraint.

The first-order KKT necessary condition on a regular local maximum $s^{\star}$ is that

$$
\begin{aligned}
\mathbf{0} & =\left(\nabla_{\boldsymbol{s}} L\right)\left(\boldsymbol{s}^{\star}, \lambda^{\star}, \boldsymbol{\mu}^{\star}, \boldsymbol{\nu}^{\star}\right) \\
& =-z+\lambda^{\star} s^{\star}-\mu^{\star}+\nu^{\star},
\end{aligned}
$$

where $\mu_{m}^{\star}>0$ only if $s_{m}^{\star}=0$ and $\nu_{m}^{\star}>0$ only if $s_{m}^{\star}=$ $\delta$. Notice that one of $\mu_{m}^{\star}$ or $\nu_{m}^{\star}$ must be zero because they correspond to mutually exclusive constraints. The second-order KKT necessary condition on a regular local maximum is that

$$
\begin{aligned}
0 & \leq y^{T}\left(\nabla_{s}^{2} L\right)\left(s^{\star}, \lambda^{\star}, \mu^{\star}, \nu^{\star}\right) y \\
& =\lambda^{\star} y^{T} y
\end{aligned}
$$

for every vector $\boldsymbol{y}$ in the subspace of first-order feasible variations. This subspace is non-trivial, so $\lambda^{\star} \geq 0$.

Solve Equation (1) to obtain

$$
\lambda^{\star} s^{\star}=z+\mu^{\star}-\nu^{\star}
$$

Whenever $\mu_{m}^{\star}>0$, both $s_{m}^{\star}=0$ and $\nu_{m}^{\star}=0$. This combination is impossible because $z_{m} \geq 0$. Therefore, we may eliminate $\mu^{\star}$ to reach

$$
\lambda^{\star} s^{\star}=z-\nu^{\star}
$$

The cases $\lambda^{\star}=0$ and $\lambda^{\star}>0$ require separate consideration.

If $\lambda^{\star}=0$, it is clear that $\nu^{\star}=z$. Since $\nu_{m}^{\star}>0$ only if $s_{m}^{\star}=\delta$, we must have $s_{m}^{\star}=\delta$ whenever $z_{m}>0$. Suppose that $k$ components of $\boldsymbol{s}^{\star}$ equal $\delta$. The remaining $(d-k)$ components are not uniquely determined by the optimization problem. From the many solutions, we choose one such that

$$
s_{m}^{\star}=\sqrt{\frac{c-k \delta^{2}}{d-k}} \quad \text { for } m \text { where } z_{m}=0 \text {. }
$$

This formula ensures that $s^{\star}$ has the correct norm and that none of its entries exceeds $\delta$.

When $\lambda^{\star}>0$, the solution has the form

$$
\boldsymbol{s}^{\star}=[\gamma \boldsymbol{z}]_{\delta}
$$

where $\gamma$ is positive and the operator $[\cdot]_{\delta}$ truncates to $\delta$ components of its argument that exceed $\delta$. It is clear that the largest components of $z$ are all truncated at the same time. We only need to determine which components these are.

To that end, observe that $\gamma \mapsto\left\|[\gamma z]_{\delta}\right\|_{2}$ is a. strictly increasing function on $\left[0, \delta / z_{\min }\right]$, where $z_{\min }$ is the least positive component of $\boldsymbol{z}$. For at most one value of $\gamma$, therefore, does the vector $[\gamma z]_{\delta}$ have norm $\sqrt{c}$. If this norm value were not attained, then $\lambda^{\star}$ would equal zero. Let $k$ be the number of entries of $s^{\star}$ that equal $\delta$, and suppose that $\mathscr{M}$ indexes the remaining $(d-k)$ components. Then

$$
c=\left\|s^{\star}\right\|_{2}^{2}=k \delta^{2}+\gamma^{2} \sum_{m \in \mathscr{M}}\left|z_{m}\right|^{2} .
$$

Recall that $\gamma$ is positive. Therefore, is impossible that $k \delta^{2}>c$. When $k \delta^{2}=c$, it follows that $z_{m}=0$ for each $m$ in $\mathscr{M}$. Otherwise, $z_{m}$ must be non-zero for some $m$ in $\mathscr{M}$. Then the value of $\gamma$ must be

$$
\gamma=\sqrt{\frac{c-k \delta^{2}}{\sum_{m \in \mathscr{M}}\left|z_{m}\right|^{2}}}
$$

\section{NUMERICAL EXPERIMENTS}

Let us demonstrate that alternating projection can indeed produce tight frames whose columns have specified PAR and specified norm. The experiments all begin with the initial $3 \times 6$ matrix

$$
\left[\begin{array}{rrc}
.0748+.3609 \mathrm{i} & .0392+.4558 \mathrm{i} & .5648+.3635 \mathrm{i} \\
.5861-.0570 \mathrm{i} & -.2029+.8024 \mathrm{i} & -.5240+.4759 \mathrm{i} \\
-.7112+.1076 \mathrm{i} & -.2622-.1921 \mathrm{i} & -.1662+.1416 \mathrm{i} \\
-.2567+.4463 \mathrm{i} & .7064+.6193 \mathrm{i} & .1586+.6825 \mathrm{i} \\
-.1806-.1015 \mathrm{i} & -.1946-.1889 \mathrm{i} & .5080+.0226 \mathrm{i} \\
.0202+.8316 \mathrm{i} & .0393-.2060 \mathrm{i} & .2819+.4135 \mathrm{i}
\end{array}\right] .
$$

The respective PAR values of its columns are 1.5521 , $2.0551,1.5034,2.0760,2.6475$ and 1.4730 . 
Unimodular tight frames are probably the most interesting example of frames with low PAR. Every entry of a unimodular frame has an identical modulus, and so the PAR of each column equals one. Let us apply the algorithm to calculate a unit-norm, unimodular tight frame.

$$
\left[\begin{array}{ccc}
.1345+.5615 \mathrm{i} & .1672+.5526 \mathrm{i} & .4439+.3692 \mathrm{i} \\
.5410-.2017 \mathrm{i} & -.0303+.5766 \mathrm{i} & -.5115+.2679 \mathrm{i} \\
-.5768+.0252 \mathrm{i} & -.2777-.5062 \mathrm{i} & -.2303+.5294 \mathrm{i} \\
-.3358+.4696 \mathrm{i} & .4737+.3300 \mathrm{i} & .0944+.5696 \mathrm{i} \\
-.5432-.1956 \mathrm{i} & -.3689-.4442 \mathrm{i} & .5747+.0554 \mathrm{i} \\
.1258+.5635 \mathrm{i} & -.0088-.5773 \mathrm{i} & .4132+.4033 \mathrm{i}
\end{array}\right] .
$$

Indeed, each of the columns has unit PAR, and the singular values of the matrix are identical to eight decimal places. The calculation required 78 iterations lasting 0.1902 seconds.

Alternating projection can also compute tight frames whose columns have unit PAR but do not have unit norm. For example, if we request the column norms $0.75,0.75$, $1,1,1.25$ and 1.25 , the algorithm yields

$$
\left[\begin{array}{rrc}
.3054+.3070 \mathrm{i} & .1445+.4082 \mathrm{i} & .3583+.4527 \mathrm{i} \\
.4295-.0549 \mathrm{i} & .1235+.4150 \mathrm{i} & -.5597+.1418 \mathrm{i} \\
-.4228-.0936 \mathrm{i} & -.0484-.4303 \mathrm{i} & .0200+.5770 \mathrm{i} \\
-.4264+.3893 \mathrm{i} & .4252+.5831 \mathrm{i} & .3622+.6242 \mathrm{i} \\
-.5393-.2060 \mathrm{i} & -.4425-.5701 \mathrm{i} & .7165-.0863 \mathrm{i} \\
.2585+.5162 \mathrm{i} & -.2894-.6611 \mathrm{i} & .1291+.7101 \mathrm{i}
\end{array}\right]
$$

One can check that the column norms, PAR and singular values all satisfy the design requirements to eight or more decimal places. The computation took 84 iterations over 0.1973 seconds.

Less stringent constraints on the PAR pose even less trouble. For example, we might like to construct a tight frame whose PAR is bounded by two and whose columns have norms $0.75,0.75,1,1,1.25$ and 1.25 . Here it is.

$$
\left.\begin{array}{rrc}
.0617+.1320 \mathrm{i} & .0184+.2764 \mathrm{i} & .4299+.3593 \mathrm{i} \\
.4256-.1031 \mathrm{i} & -.0558+.5938 \mathrm{i} & -.5920+.4974 \mathrm{i} \\
-.5912+.0025 \mathrm{i} & -.1304-.3363 \mathrm{i} & -.0807+.2857 \mathrm{i} \\
-.1382+.2511 \mathrm{i} & .6847+.7436 \mathrm{i} & .2933+.6939 \mathrm{i} \\
-.4306-.2650 \mathrm{i} & -.2095-.3072 \mathrm{i} & .7317+.0928 \mathrm{i} \\
.0852+.8093 \mathrm{i} & -.3504-.5289 \mathrm{i} & .2918+.6048 \mathrm{i}
\end{array}\right] .
$$

The computer worked for 0.0886 seconds, during which it performed 49 iterations. As usual, the singular values match to eight decimal places. It is interesting to observe that the frame exceeds the design specifications. The respective PAR values of its columns are 1.8640, 1.8971, $1.7939,1.9867,1.9618$ and 1.0897 .

\section{CONCLUSIONS AND FURTHER WORK}

We have proposed a method for constructing optimal CDMA signature sequences that satisfy a constraint on the peak-to-average power ratio. The algorithm is based on an alternating projection between a spectral constraint and the PAR constraint.

The flexibility of the alternating projection approach suggests that it may be able to address other constraints. This is indeed the case. For example, a straightforward modification of this algorithm can construct sequences whose Fourier transform is nearly unimodular, which is the frequency-domain analog of low peak-to-average power ratio. For some other applications, see the paper [10].

In further work, it would be interesting to develop a method for finding signatures for synchronous CDMA systems operating in the presence of colored noise. Extensions to asynchronous systems, and systems with multipath interference, also merit further investigation.

\section{REFERENCES}

[1] M. Rupf and J. L. Massey, "Optimum sequence multisets for synchronous code-division multiple-access channels," IEEE Trans. IT, vol. 40, no. 4, pp. 1261-1266, July 1994.

[2] Pramod Viswanath and Venkat Anantharam, "Optimal sequences and sum capacity of synchronous CDMA systems," IEEE Trans. IT, vol. 45, no. 6, pp. 1984-1991, Sept. 1999.

[3] P. Viswanath and A. Anantharam, "Optimal sequences for CDMA under colored noise: A Schur-saddle function property," IEEE Trans. IT, vol. 48, no. 6, pp. 1295-1318, June 2002.

[4] V. K. N. Lau, "On the analysis of peak-to-average ratio (PAR) for IS95 and CDMA2000d systems," IEEE Trans. on Vehicular Tech., vol. 49, no. 6, pp. 2174-2188, Nov. 2000.

[5] Sennur Ulukus and Roy D. Yates, "Iterative construction of optimum signature sequence sets in synchronous CDMA systems," IEEE Trans. IT, vol. 47, no. 5, pp. 1989-1998, 2001.

[6] C. Rose, "CDMA codeword optimization: Interference avoidance and convergence via class warfare," IEEE Trans. IT, vol. 47 , no. 6 , pp. 2368-2382, 2001.

[7] S. Ulukus and A. Yener, "Iterative joint optimization of CDMA signature sequences and receiver filters," in Conference on Information Sciences and Systems, Princeton Univ., Mar. 2002.

[8] J. A. Tropp, R. W. Heath Jr., and T. Strohmer, "An alternating projection algorithm for constructing quasi-orthogonal CDMA signature sequences," in Proc. Int. Symp. on Info. Theo., 2003.

[9] Moody T. Chu, "Constructing a Hermitian matrix from its diagonal entries and eigenvalues," SIAM J. Matrix Anal. Appl., vol. 16, no. 1, pp. 207-217, Jan. 1995.

[10] J. A. Tropp, I. S. Dhillon, R. W. Heath Jr., and T. Strohmer, "Designing structured tight frames via alternating projection," Preprint, also available as an ICES Report, The University of Texas at Austin, December, 2003.

[11] Roger A. Horn and Charles R. Johnson, Matrix Analysis, Cambridge University Press, 1985.

[12] R. T. Rockafellar, Convex Analysis, Princeton Univ. Press, 1970. 